\title{
Prevalence of Troglodytella abrassarti Brumpt and Joyeux, 1912 in Wild Chimpanzees (Pan troglodytes schweinfurthii) at Mahale Mountains National Park in Western Tanzania
}

Author(s): Taranjit Kaur , Jatinder Singh , and David S. Lindsay

Source: Journal of Parasitology, 96(1):209-210. 2010.

Published By: American Society of Parasitologists

DOI: http://dx.doi.org/10.1645/GE-2266.1

URL: http://www.bioone.org/doi/full/10.1645/GE-2266.1

BioOne (www.bioone.org) is a nonprofit, online aggregation of core research in the biological, ecological, and environmental sciences. BioOne provides a sustainable online platform for over 170 journals and books published by nonprofit societies, associations, museums, institutions, and presses.

Your use of this PDF, the BioOne Web site, and all posted and associated content indicates your acceptance of BioOne's Terms of Use, available at www.bioone.org/page/terms of use.

Usage of BioOne content is strictly limited to personal, educational, and non-commercial use. Commercial inquiries or rights and permissions requests should be directed to the individual publisher as copyright holder. 


\title{
Prevalence of Troglodytella abrassarti Brumpt and Joyeux, 1912 in Wild Chimpanzees (Pan troglodytes schweinfurthii) at Mahale Mountains National Park in Western Tanzania
}

\author{
Taranjit Kaur, Jatinder Singh, and David S. Lindsay, Department of Biomedical Sciences and Pathobiology, Virginia Tech University, \\ Blacksburg, Virginia 24061.e-mail: tsy/vina@vt.edu
}

\begin{abstract}
We examined stool samples for trophozoites of the entodiniomorphid ciliate Troglodytella abrassarti Brumpt and Joyeux, 1912, from a habituated group of chimpanzees (Pan troglodytes schweinfurthii) at Mahale Mountains National Park in western Tanzania. In our study, fresh fecal samples from identified individuals were collected immediately after defecation and fixed in $10 \%$ formalin. In total, 52 samples from 38 chimpanzees ( $61 \%$ of 62 chimpanzees in the group) were examined using a direct smear method. A stool sample from an individual collection date from an individual chimpanzee was examined up to 3 separate times before it was called negative. Forty-eight (92\%) of the 52 samples were positive, and stools from 37 (97\%) of the 38 chimpanzees were positive for trophozoites of $T$. abrassarti. The high prevalence of $T$. abrassarti in these chimpanzees is consistent with previous reports of this organism in chimpanzees.
\end{abstract}

Troglodytella abrassarti Brumpt and Joyeux, 1912 is an entodiniomorphid ciliate often observed in the feces of primates (Goussard et al., 1983; O’Donoghue et al., 1993). It lives in the large intestine of its host and has been suggested to be beneficial in digesting fibrous material (Irbis et al., 2008). Recent studies using the small subunit rRNA gene and internal transcribed spacer rDNA spacer region have demonstrated that the organisms from gorillas (Gorilla gorilla gorilla) and chimpanzees are the same (Modry et al., 2009). The organism is usually considered a commensal, but it has been associated with diarrhea in siamangs (Hylobates syndactylus) from a zoo in Australia (O’Donoghue et al., 1993).

Our study subjects were chimpanzees, Pan troglodytes schweinfurthii, at the Mahale Mountains National Park in western Tanzania. Beginning in March 1966, habituation efforts were initiated by researchers from Japan to attract chimpanzees from 1 unit-group, referred to as the M-Group, allowing human observers to approach them in their natural habitat (Nishida, 1990). The purpose of the present study was to examine stools from chimpanzees in this population for the prevalence of trophozoites of T. abrassarti.

We examined 52 stool samples from 38 individual chimpanzees $(61 \%)$ from M-Group ( $\mathrm{n}=62$ chimpanzees). Males (14) and female (24) chimpanzees were sampled from 4 age groups, i.e., 2 infants $(0-3 \mathrm{yr}), 6$ juveniles ( $4-8 \mathrm{yr}), 9$ adolescents (9-14 yr), and 21 adults ( $\geq 15 \mathrm{yr}$ ). A single sample was examined from 26 of the chimpanzees, samples from 10 chimpanzees were examined from 2 different collection days, and samples from 2 chimpanzees were examined from 3 different collection days. Samples were collected from June and August 2005, and fresh stool samples from identified individuals were collected immediately after defecation. Stool consistencies (loose $=$ soft or not well formed; firm $=$ well formed) were recorded. Only that part of fecal material not in contact with the forest floor was collected. A portion of the sample was used to examine for the presence of occult blood (Hemoccult ${ }^{\circledR}$ Fecal Occult Blood Test; Beckman Coulter, Fullerton, California). Another portion of the stool sample was placed in a weigh boat and thoroughly mixed with a clean wooden applicator stick. Approximately, $10 \mathrm{~g}$ of stool were placed in a $50-\mathrm{ml}$ sterile tube containing $10 \mathrm{ml}$ of $10 \%$ buffered formalin solution and mixed vigorously by shaking. Samples were stored at $30 \mathrm{C}$ until shipped to the Department of Biomedical Sciences and Pathobiology (DBSP), Virginia-Maryland Regional College of Veterinary Medicine, Blacksburg, Virginia. An approximate 8-ml aliquot of the sample was placed in a $15-\mathrm{ml}$ plastic-screw-capped centrifuge tube and kept at $4 \mathrm{C}$ until examined.

The stool solution was vigorously shaken by hand, and a drop was placed on a glass microscope slide and covered with a $22-\mathrm{mm}^{2}$ coverslip.

DOI: $10.1645 / G E-2266.1$
The entire coverslip was scanned at $\times 10$ using an Olympus BX60 microscope (Olympus America Inc., Center Valley, Pennsylvania) by the same observer (DSL). The observer was blind to the identity of each stool sample. Identification of $T$. abrassarti trophozoites was confirmed by using the $\times 40$ objective of the microscope to see structural features consistent with this organism (O'Donoghue et al., 1993). The length of $T$. abrassarti trophozoites was determined using a calibrated ocular micrometer and a $\times 40$ objective. Additional drops of stool suspension were examined up to a maximum of 3 times on a sample from an individual subject before a subject was considered negative.

Forty-eight $(92 \%)$ of the 52 stool samples contained trophozoites (Figs. 1-3) of T. abrassarti. Formalin-fixed trophozoites $(\mathrm{N}=47)$ of $T$. abrassarti measured $150 \pm 24 \mu \mathrm{m}$ (range $=93$ to $214 \mu \mathrm{m})$. The adoral ciliature, somatic ciliature (Fig. 1), and L-shaped macronucleus (Fig. 2) were not always readily visible, but trophozoites were easily recognized because of their large size and the posterior protuberance (Fig. 3). Twenty-five $(96 \%)$ of the 26 stool samples from chimpanzees examined only on 1 occasion were positive for trophozoites of T. abrassarti. The single sample negative for trophozoites of $T$. abrassarti was from an adult female. Stool samples from $8(80 \%)$ of 10 chimpanzees examined on 2 occasions were positive for trophozoites of $T$. abrassarti on both examination dates. Samples from 1 chimpanzee were negative on the first examination date but positive on the second examination date, and samples from another chimpanzee were positive on the first examination date and negative on the second. Trophozoites of T. abrassarti were detected in both chimpanzees that were examined on 3 separate occasions. Stool samples from 1 of these chimpanzees were positive on the first examination, negative on the second, and positive on the third, whereas all samples from the second chimpanzee that was examined on 3 occasions were consistently positive.

Stool consistency was reported on 22 of the 52 samples. Of the 22 samples, 21 contained trophozoites of T. abrassarti, of which 3 of the 21 were loose; 1 of these 3 stool samples was positive for occult blood. The other 18 of the 21 positive samples (86\%) were firm, of which 8 (of the 18 , or $44 \%$ ) were positive for occult blood. The 1 stool sample that was negative for trophozoites of T. abrassarti was firm and occult blood negative.

The trophozoites of T. abrassarti in the present study (Figs. 1-3) ranged from 93 to $214 \mu \mathrm{m}$ in size and averaged $150 \mu \mathrm{m}$ in length. This is larger than the average length of $134 \mu \mathrm{m}$ (range 100 to $180 \mu \mathrm{m} ; \mathrm{N}=50$ ) for trophozoites of T. abrassarti reported by O'Donoghue et al. (1993) from captive siamangs (H. syndactylus) in an Australian zoo. It is possible that these differences in length are because of the different methods used to examine samples. O'Donoghue et al. (1993) examined samples that had been processed by protargol impregnation, and we examined formalin-

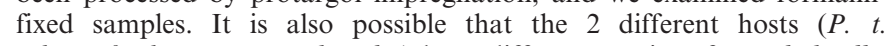
schweinfurthii vs. H. syndactylus) have different species of Troglodytella. Additionally, the wide range in measurements of trophozoites $(93$ to $214 \mu \mathrm{m}$ ) of T. abrassarti suggests that more than 1 species of Troglodytella may be present in chimpanzees. Further genetic analysis is needed to clarify the taxonomy of this group of entodiniomorphid ciliates.

There have been a few studies examining the prevalence of $T$. abrassarti in chimpanzees in their natural habitat (File et al., 1976; Ashford et al., 2000; Murray et al., 2000; Krief et al., 2005; Muehlenbein, 2005). Trophozoites of T. abrassarti were observed in $24(75 \%)$ of 32 wild chimpanzees from Gombe National Park, Tanzania, using the formalinether concentration technique (File et al., 1976). Troglodytella abrassarti trophozoites were observed in chimpanzees from all age groups (infant, juvenile, adolescent, and adult) examined (File et al., 1976). In another study of chimpanzees and baboons, Papio cynocephalus anubis, from Gombe National Park, Tanzania, trophozoites of $T$. abrassarti were 


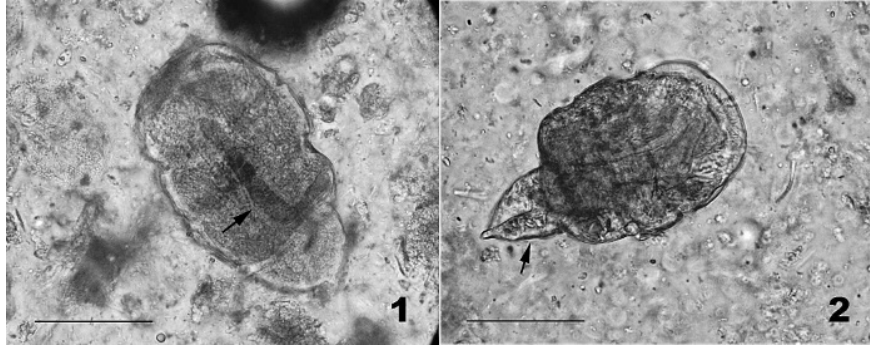

Figure 1. Trophozoite of Troglodytella abrassarti in nonstained fecal smears. Trophozoite demonstrates an L-shaped macronucleus (arrow). Note the somatic ciliature is not readily visible. Bar $=50 \mu \mathrm{m}$.

Figure 2. Trophozoite of Troglodytella abrassarti in nonstained fecal smears. The trophozoite demonstrates a prominent posterior protuberance (arrow). Bar $=50 \mu \mathrm{m}$

observed in $3(14 \%)$ of 20 samples from chimpanzees and none of 35 samples from baboons scrutinized using the direct smear (Murray et al., 2000). Ashford et al. (2000) examined 123 stool samples from 45 chimpanzees from the Kanyawara area of the Kibale National Park, Uganda, and found that $98 \%$ of the 45 stool specimens from individual chimpanzees sampled were positive for trophozoites of $T$. abrassarti. They reported that $91 \%$ of the 123 total samples were positive for trophozoites of T. abrassarti (Ashford et al., 2000). In another study of chimpanzees from the Kanyawara area of the Kibale National Park, Uganda, Krief et al. (2005) found that $111(61 \%)$ of stool samples from 38 chimpanzees were positive for trophozoites of $T$. abrassarti during the dry season, and $50(77 \%)$ of 65 stool samples collected during the rainy season were positive for trophozoites of $T$. abrassarti. They based their results on examination of $200 \mu \mathrm{l}$ of formalin-fixed stool sample from each animal Muehlenbein (2005) conducted a study on 121 stool samples from 37 male chimpanzees from the Ngogo area of Kibale National Park, Uganda, using the formalin-ethyl acetate sedimentation technique. They found that $97 \%$ of the 37 chimpanzees were positive for trophozoites of $T$. abrassarti, while $84 \%$ of the 121 total samples from these chimpanzees were positive for trophozoites of $T$. abrassarti. Our study is in agreement with the findings of Ashford et al. (2000) and Muehlenbein (2005), indicating that chimpanzees are frequently hosts for trophozoites of $T$. abrassarti. It should be noted that Murray et al. (2000) were not focusing on the prevalence of $T$. abrassarti in their study and used only a direct smear to detect infections; this may explain the lower prevalence of $20 \%$ (3 of 20) of positive chimpanzees that they observed.

Methods that rely on fecal flotation are unlikely to detect trophozoites of T. abrassarti as demonstrated by Krief et al. (2005). They examined 239 fecal samples from 38 chimpanzees from the Kibale National Park, Uganda, by the McMaster method using $\mathrm{MgSO}_{4}$, and found no positive samples for trophozoites of $T$. abrassarti (Krief et al., 2005). In the present study, the only chimpanzee that had no detectable trophozoites of $T$. abrassarti in its stool sample was examined just once. We showed that stool samples from different collection days revealed $100 \%$ prevalence of trophozoites of $T$. abrassarti in the 12 chimpanzees that were examined on multiple occasions. Because of these findings, we recommend that 3 stool samples should be found to be negative from an individual chimpanzee before it can be considered truly negative for trophozoites of T. abrassarti.

Because we did not examine stool samples for other pathogens, we can make no definitive comments on the association of T. abrassarti infection and the presence of blood in the stool or loose stool in the chimpanzees examined. However, our results do suggest that the presence of trophozoites of $T$. abrassarti is not associated with blood in the stool or loose stools.

This study was funded in part by NSF grant 0238069 to T.K. Any opinions, findings, and conclusions or recommendations expressed in this materials are those of the author(s) and do not necessarily reflect the views of NSF.

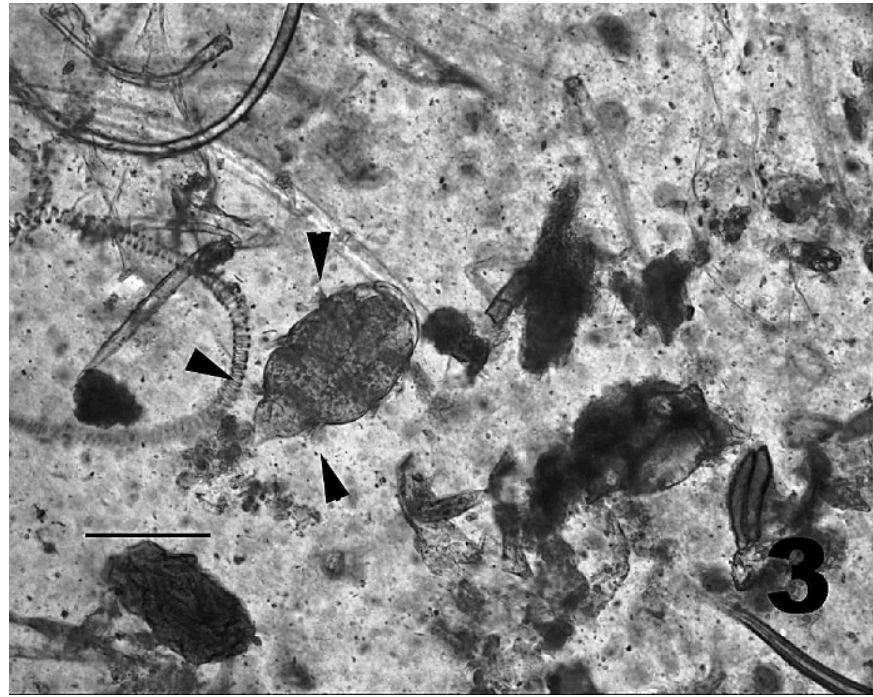

FIgURE 3. Trophozoite of Troglodytella abrassarti in nonstained fecal smear. The arrowheads point to somatic ciliature that is visible on this specimen. Bar $=100 \mu \mathrm{m}$.

\section{LITERATURE CITED}

Ashford, R. W., G. D. Reid, and R. W. Wrangham. 2000. Intestinal parasites of the chimpanzee Pan troglodytes in Kibale Forest, Uganda. Annals of Tropical Medicine Parasitology 94: 173-179.

File, S. K., W. C. McGrew, and C. E. Tutin. 1976. The intestinal parasites of a community of feral chimpanzees, Pan troglodytes schweinfurthii. Journal of Parasitology 62: 259-261.

Goussard, B., J. Y. Collet, Y. Garin, C. E. Tutin, and M. Fernandez. 1983. The intestinal entodiniomorph ciliates of wild lowland gorillas (Gorilla gorilla gorilla) in Gabon, West Africa. Journal of Medical Primatology 12: 239-249.

Irbis, C., R. Garriga, A. Kabasawa, and K. Ushida. 2008. Phylogenetic analysis of Troglodytella abrassarti isolated from Chimpanzees (Pan troglodytes verus) in the wild and in captivity. Journal of General and Applied Microbiology 54: 409-413.

Krief, S., M. A. Huffman, T. Sévenet, J. Guillot, C. Bories, C. M. HLADIK, and R. W. WRangham. 2005. Noninvasive monitoring of the health of Pan troglodytes schweinfurthii in the Kibale National Park, Uganda. International Journal of Primatology 26: 467-490.

Modry, D., K. J. Petrzelková, K. Pomajbíková, T. Tokiwa, T. Krízek, S. Imai, P. Vallo, I. Pofousová, and J. Slapeta. 2009. The occurrence and ape-to-ape transmission of the entodiniomorphid ciliate Troglodytella abrassarti in captive gorillas. Journal of Eukaryotic Microbiology 56: 83-87.

Muehlendein, M. P. 2005. Parasitological analyses of the male chimpanzees (Pan troglodytes schweinfurthii) at Ngogo, Kibale National Park, Uganda. American Journal of Primatology 65: 167179.

Murray, S., C. Stem, B. Boudreau, and J. Goodall. 2000. Intestinal parasites of baboons (Papio cynocephalus anubis) and chimpanzees (Pan troglodytes) in Gombe National Park. Journal of Zoo and Wildlife Medicine 31: 176-178.

NishidA, T. 1990. The chimpanzees of the Mahale Mountains: Sexual and life history strategies. University of Tokyo Press, Tokyo, Japan, $20 \mathrm{p}$.

O’Donoghue, P.J., R. B. Gasser, and A. Tribe. 1993. New host record for the entodiniomorphid ciliate, Troglodytella abrassarti, from siamangs (Hylobates syndactylus). International Journal for Parasitology 23: 415-418. 